\section{Atributos da atenção primária na assistência à saúde da criança: avaliação dos cuidadores}

\section{Aspects of primary care for children: an evaluation of care-givers}

Claudia Danyella Alves Leão 1

Antônio Prates Caldeira 2

Mônica Maria Celestina de Oliveira 3

${ }^{1}$ Universidade Estadual de Montes Claros. Montes Claros, MG, Brasil. CEP: 39.401-089. E-mail: claudiadanyella@hotmail.com

2 Departamento de Saúde da Mulher e da Criança da Universidade Estadual de Montes Claros. Montes Claros, MG, Brasil.

${ }^{3}$ Universidade Federal do Rio Grande do Sul. Porto Alegre, RS, Brasil.

\title{
Resumo
}

Objetivos: avaliar os atributos da atenção primária à saúde (APS), na assistência à saúde infantil ofertada pelas equipes da Estratégia Saúde da Família (ESF) em comparação a outros serviços de atenção à saúde da criança em Montes Claros $(M G)$.

Métodos: pesquisa transversal, descritiva, de abordagem quantitativa. Para coleta de dados utilizou-se questionário validado no Brasil denominado de Instrumento de Avaliação da Atenção Primária na versão infantil (PCATool infantil). $O$ instrumento foi aplicado aos cuidadores das crianças residentes e cadastradas nas áreas de abrangência das equipes de saúde da família. Foram atribuidos escores às oito dimensões da APS.

Resultados: apenas duas dimensões apresentaram valores satisfatórios para as crianças que utilizavam a ESF como fonte regular de atenção à saúde. Em geral os dados não apresentam diferenças significativas entre as crianças que utilizam a ESF para as que utilizam outros tipos de serviço, exceto para a integralidade (serviços complementares disponíveis), orientação familiar e comunitária. O escore geral da APS mostrou valor próximo ao ideal entre as crianças que utilizavam a ESF como fonte de atenção à saúde e superior ao escore atribuido pelos cuidadores que utilizavam outros serviços.

Conclusão: fidelidade aos atributos da APS foram mais observadas nas equipes da ESF, no entanto, existem ainda alguns aspectos que precisam ser melhoradas.

Palavras-chave Atenção primária à saúde, Programa saúde da família, Avaliação de serviços 


\section{Introdução}

O século XX foi marcado pelo desenvolvimento de diversas experiências de modelos assistenciais em saúde em todo o mundo que serviram de base para o estabelecimento dos princípios e diretrizes propostos na Declaração de Alma Ata, que foi formulada por ocasião da Conferência Internacional sobre Cuidados Primários de Saúde. ${ }^{1}$ O fortalecimento da Atenção Primária à Saúde (APS) foi estabelecido, a partir de então, como a principal estratégia para organização dos serviços públicos de saúde. ${ }^{1,2}$

No Brasil, várias propostas de modelo de APS, como o da medicina geral e comunitária, o Programa de Médicos de Família de Niterói, as Ações Programáticas em Saúde e o Movimento em Defesa da Vida, foram formuladas como alternativas ao modelo de saúde vigente. ${ }^{3}$ Todas essas propostas foram importantes e exitosas, porém não resultaram na transformação do modelo assistencial vigente. Assim, ocorreram reformulações sucessivas do modelo assistencial, até o modelo da Estratégia Saúde da Família (ESF) ser instituído no âmbito nacional. 4

Em 2006, o Ministério da Saúde editou a Política de Atenção Básica, revisando as diretrizes e normas para a organização da ESF. Através de portaria específica, ampliou o escopo das ações da APS, colocando as unidades de saúde da família como portas de entrada preferenciais para o Sistema Único de Saúde (SUS) e como estratégia de organização dos sistemas locais de saúde. Propôs, dessa forma, uniformizar o processo de trabalho das equipes e reconheceu a ESF, crescente em todo o país, como modelo substitutivo em relação à rede de atenção básica. 5

A ampliação da rede de atenção ambulatorial parece não ter sido um fenômeno apenas no Brasil e a percepção de que o simples incremento dessa rede de assistência não apresenta impacto significativo sobre muitos dos indicadores de saúde levou a Organização Panamericana da Saúde a elaborar uma proposta de renovação da Atenção Primária nas Américas. ${ }^{6}$ Existe o propósito claro de fortalecimento da APS, com ênfase na incorporação de valores, princípios e elementos próprios da atenção primária efetiva, fatoque representa um avanço em relação ao incentivo de incremento meramente numérico, sem compromisso com a qualidade.

Pode-se considerar que melhores resultados dos serviços de atenção primária à saúde serão alcançados a partir do conhecimento e operacionalização de seus princípios ordenadores. Evidências vêm se acumulando há décadas de que sistemas de serviços de saúde que se organizam a partir de uma atenção primária à saúde, estruturada em conformidade com os seus atributos ordenadores, são mais eficazes e de maior qualidade. ${ }^{7-11}$

Starfield 12 definiu quatro atributos essenciais da APS: primeiro contato, continuidade ou longitudinalidade, integralidade e coordenação e dois atributos derivados: centralização familiar e orientação comunitária $\mathrm{O}$ primeiro contato implica em acessibilidade e uso de serviços para cada novo problema ou para acompanhamento rotineiro de saúde. A longitudinalidade é definida como a existência do aporte regular de cuidados pela equipe de saúde e seu uso consistente ao longo do tempo em um ambiente de relação mútua entre equipe de saúde, indivíduos e famílias. A integralidade consiste na prestação, pela equipe de saúde, de um conjunto de serviços que atendam às necessidades mais comuns da população adscrita, a responsabilização por outros pontos de atenção à saúde e o reconhecimento adequado dos problemas que causam as doenças; implica em oferta de serviços preventivos e curativos e a garantia dos diversos tipos de serviços. A coordenação é definida pela capacidade de garantir a continuidade da atenção no interior da rede de serviços. A centralização familiar é um atributo derivado que implica em considerar a família como o sujeito da atenção. A orientação comunitária pressupõe o reconhecimento das necessidades familiares em função do contexto físico, econômico, social e cultural em que vivem. 12

Estudos avaliativos na área de atenção primária deveriam certificar-se da aderência aos princípios descritos, antes da análise de indicadores. Para alguns autores, é o grau de comprometimento do sistema e dos serviços de saúde com os princípios da APS que asseguram maior efetividade da atenção oferecida. ${ }^{13}$ Um sistema de saúde com forte referencial na Atenção Primária à Saúde é mais efetivo, mais satisfatório para a população, tem menores custos e é mais equitativo - mesmo em contextos de grande iniquidade social. ${ }^{14}$

A literatura registra instrumentos de avaliação da APS, com ênfase na aferição da presença e extensão dos atributos essenciais e derivados da atenção primária à saúde. 15,16 O Primary Care Assessment Tool (PCATool) já foi validado no Brasil e possibilita avaliar o grau de orientação à APS através dos seus atributos, podendo ser aplicado a profissionais de saúde ou usuários e dirigido às ações de saúde de adultos ou crianças (em versões distintas), refletindo a experiência de diferentes grupos em relação ao serviço de saúde. 17

O objetivo do presente estudo foi comparar os 
atributos da atenção primária, na assistência à saúde da criança nas equipes de saúde da família com a de outros serviços de atenção infantil em um município do Estado de Minas Gerais, a partir da avaliação dos cuidadores.

\section{Métodos}

Trata-se de um estudo transversal, de abordagem quantitativa, realizado no município de Montes Claros, ao Norte do Estado de Minas Gerais. A cidade possui população local estimada em 361.971 habitantes. Quanto aos serviços de atenção primária, a cidade contava à época da coleta de dados (segundo semestre de 2009) com 49 equipes de saúde da família, (44 localizados na zona urbana e 05 na zona rural), 15 unidades de saúde com o Programa de Agentes Comunitários de Saúde (PACS) e 15 centros de saúde, sendo estes últimos responsáveis pela assistência da população fora da área de cobertura das ESF.

A população estudada foi constituída por cuidadores de crianças de zero a dois anos de idade, residentes e cadastradas nas áreas de abrangência das equipes da ESF do município. Foram incluídas apenas as equipes localizadas na zona urbana, que atuavam há no mínimo um ano após o início da coleta de dados, pelo maior contato com a população e os serviços de saúde. Após a aplicação desses critérios, população infantil identificada foi de 3647 crianças na faixa etária de zero a dois anos de idade, distribuídas em 43 equipes (uma das equipes da área urbana estava instalada há menos de um ano).

As famílias das crianças foram selecionadas através de amostragem aleatória simples. Inicialmente atribuiu-se um número a cada família de crianças na faixa etária referida. Em seguida procedeu-se o sorteio a partir da tabela de números aleatórios do número de famílias definido pelo cálculo amostral. Para as famílias que possuíam mais de uma criança com idade de zero a dois anos, apenas informações referentes à criança mais velha foram coletados. A identificação das famílias foi realizada a partir do cadastro das mesmas nas unidades da ESF. O cálculo definiu um tamanho da amostra de 350 crianças, considerando-se um erro amostral de $5 \%$ e nível de confiança de $95 \%$ e admitindo-se uma prevalência dde $50 \%$ do evento estudado (ter a Unidade de Saúde da Família como principal fonte de cuidado da criança). A seleção foi feita por sorteio da população adscrita em todas as unidades e o número de crianças/famílias sorteadas foi proporcional ao cadastro de cada uma das equipes elegíveis para o estudo.
A coleta de dados foi realizada utilizando um, instrumento elaborado e validado no Brasil, por Harzheim et al., 17 que afere a extensão dos atributos essenciais e derivados da APS em serviços de atenção à saúde infantil. A entrevista foi realizada com o principal cuidador da criança nos domicílios.

A versão validada do instrumento possui 45 perguntas para aferição dos atributos (seis para primeiro contato, dez para continuidade ou longitudinalidade, cinco para coordenação, 14 para integralidade, seis para orientação familiar e quatro para orientação comunitária) e outras três perguntas que medem o grau de afiliação ao serviço de saúde. As respostas são do tipo Likert, com intervalo de um a quatro para cada atributo. As respostas possíveis para cada um dos itens foram: "com certeza sim" (valor=4), "provavelmente sim" (valor=3), "provavelmente não" (valor=2), "com certeza não" (valor=1) e "não sei / não lembro" (valor=9).

O tipo de serviço preferencial para atendimento a saúde da criança, foi definido pelo cuidador com base na aplicação de três perguntas iniciais contidas no PCATool18: 1) Há um médico/enfermeiro ou serviço de saúde onde você geralmente leva a criança quando ele(a) está doente ou quando precisa de algum conselho sobre a saúde dele(a)? 2) Há um médico/enfermeiro ou serviço de saúde que conhece melhor a criança como pessoa? e 3) Há um médico/enfermeiro ou serviço de saúde que é mais responsável pelo atendimento de saúde da criança?

Quando o cuidador referia dois serviços de saúde diferentes, era selecionado o serviço que havia sido referido duas vezes. Caso o cuidador referisse três serviços, era identificado o serviço informado na primeira pergunta. Quando houvesse resposta negativa para as três perguntas (quando não se informava nenhum serviço, selecionava-se o serviço que a criança havia sido consultada pela última vez. Em seguida, os serviços selecionados foram categorizados segundo o tipo "unidade de saúde da família" e "outros serviços" (outro tipo de serviço de saúde).

O grau de afiliação do cuidador ao serviço de saúde/profissional de saúde, ou seja, a frequência na qual reconhece o serviço ou o profissional de saúde como referência para a assistência à saúde da criança foi calculado mediante a utilização de um algoritmo estruturados de acordo com as diferentes possibilidades de respostas do cuidador às três perguntas iniciais, 18 conforme se apresenta a seguir:

Grau de afiliação $=1$ :

- Todas as respostas NÃO:

Pergunta $1=$ Pergunta $2=$ Pergunta $3=$ NÃO Grau de afiliação $=2$ :

- Uma, duas ou três respostas SIM, porém dife- 
rentes, relativas a diferentes serviços:

Pergunta $1 \neq$ Pergunta $2 \neq$ Pergunta $3 \neq$ NÃO

Grau de afiliação $=3$ :

- Duas respostas SIM iguais, relativas ao mesmo serviço:

Pergunta $1=$ Pergunta 2 ou Pergunta $1=$ Pergunta 3 ou Pergunta $2=$ Pergunta 3 e iguais a SIM

Grau de afiliação $=4$ :

- Todas as respostas SIM, todas relativas ao mesmo serviço:

Pergunta $1=$ Pergunta $2=$ Pergunta $3=$ SIM

Dados sobre as características demográficas socioeconômicas da família (idade da criança e da mãe, número de irmãos, número de pessoas na família, cuidador principal, classe econômica, escolaridade materna e paterna) foram obtidos. Outras informações como, consultas com especialistas, tempo de atendimento pelo serviço de saúde, número de consultas e motivo de escolha do serviço/modelo assistencial, foram igualmente obtidos.

Foram calculados os escores essencial (obtido através da média dos atributos essenciais e do grau de afiliação), o derivado (obtido através da média dos atributos derivados) e o escore geral da APS (valor médio dos atributos essenciais e derivados e o do grau de afiliação).18 O processamento e análise dos dados foram realizados através do software SPSS for Windows, versão 15.0.

Após a consolidação dos dados relativos a cada atributo, os valores foram transformados em uma escala contínua, variando entre zero e dez, utilizando a fórmula a seguir:

Escore ajustado $=[($ escore obtido -1$) /(4-1)] \mathrm{x}$ 10.

Valores de escores $\geq 6,6$ foram definidos como elevados e equivalentes ao valor três ou mais na escala Likert. 18 Valores $<6,6$ foram considerados baixos.

Durante a análise dos dados, buscou-se identificar as variáveis que poderiam estar relacionadas a melhor avaliação dos cuidados percebidos pelo serviço identificado como fonte regular de atenção para o cuidado de saúde das crianças. Nesse sentido, o grupo foi dicotomizado não em relação ao serviço preferencial, mas em relação ao escore atribuído a este serviço. Tal análise foi realizada através do alto escore geral da APS $(\geq 6,6)$, entre as crianças da amostra global. Inicialmente realizou-se uma análise bivariada, tomando-se todas as variáveis com nível de significância de até $25 \%(p<0,25)$. Em seguida, realizou-se uma análise de regressão múltipla (Regressão de Poisson) para definir um modelo explicativo final. A magnitude da associação. Para a comparação das médias de cada atributo entre os tipos de serviços, foi utilizado o teste "t" de Student e para análise das proporções utilizou-se do teste do qui-quadrado de Pearson. $\mathrm{O}$ nível de significância assumido foi $p<0,05$, buscando associação entre o grau de orientação (isto é, maior escore geral) para a APS e características socioeconômicas e demográficas e de utilização dos serviços de saúde. Ajustou-se um modelo de regressão de Poisson, sendo estimada a razão de prevalência e o intervalo de confiança de $95 \%$.

Foram considerados todos os preceitos éticos na condução do estudo. Os cuidadores foram esclarecidos sobre os objetivos da pesquisa e solicitados a ler e assinar o termo de consentimento livre e esclarecido. O presente estudo foi aprovado pelo Comitê de Ética em Pesquisas da Universidade Estadual de Montes Claros (Parecer 1117/2008).

\section{Resultados}

Foram registradas apenas duas recusas entre as famílias selecionadas para o estudo, sendo outras duas alocadas para reposição.

Os principais cuidadores das crianças foram as mães $(88,6 \%)$ e os avós $(5,1 \%)$. Das crianças selecionadas, $177(50,6 \%)$ eram do sexo feminino. A média de idade do grupo estudado foi de 12,7 meses $(\mathrm{DP}=6,7)$. Um total de $213(60,9 \%)$ crianças possuia irmãos, das quais $101(28,9 \%)$ possuíam dois ou mais irmãos. Observou-se que $272(77,7 \%)$ dos cuidadores identificaram a ESF com principal responsável pelo cuidado de saúde das crianças, enquanto que $78(22,3 \%)$ crianças, mesmo cadastradas no PSF, informaram outros serviços como referência.

A Tabela 1 apresenta algumas características sociodemográficas da amostra, segundo o modelo de assistência regularmente utilizado pelo cuidador da criança. Idade da mãe, número de irmãos, número de pessoas na família, número de cômodos no domicilio, classe econômica e escolaridade materna estiveram estatisticamente associados ao modelo de assistência que assistia regularmente a criança.

As características de saúde e de utilização de serviços de saúde do grupo estudado relatadas pelos cuidadores das crianças são apresentadas na Tabela 2. Observou-se um menor percentual de crianças com doenças crônicas entre aquelas assistidas regularmente pelas ESF em comparação com aquelas que frequentavam outros serviços.

Os escores dos atributos da APS para a ESF e para os outros tipos de serviço estão na Tabela 3. Ambos os modelos de atenção (ESF e outros serviços) apresentaram valores de escores satis- 
Características sociodemográficas de crianças menores de dois anos de idade, cadastradas nas equipes da Estratégia Saúde da Família, segundo o tipo de serviço referido pelo cuidador como fonte regular de atenção à saúde. Montes Claros, MG, 2009.

\begin{tabular}{|c|c|c|c|c|c|}
\hline \multirow{2}{*}{ Variáveis } & \multicolumn{2}{|c|}{ ESF } & \multicolumn{2}{|c|}{ Outros serviços } & \multirow{2}{*}{$p^{*}$} \\
\hline & \multicolumn{2}{|c|}{$\bar{x} \pm D P$} & \multicolumn{2}{|c|}{$\bar{X} \pm D P$} & \\
\hline Idade das crianças (meses) & \multicolumn{2}{|c|}{$12,8 \pm 6,7$} & \multicolumn{2}{|c|}{$12,4 \pm 6,7$} & 0,667 \\
\hline Idade das mães em anos & \multicolumn{2}{|c|}{$25,8 \pm 6,0$} & \multicolumn{2}{|c|}{$27,3 \pm 5,7$} & 0,041 \\
\hline Idade dos pais (anos) & \multicolumn{2}{|c|}{$29,6 \pm 7,6$} & \multicolumn{2}{|c|}{$30,8 \pm 6,9$} & 0,205 \\
\hline Número de irmãos & \multicolumn{2}{|c|}{$1,3 \pm 1,6$} & \multicolumn{2}{|c|}{$0,9 \pm 0,9$} & 0,018 \\
\hline Pessoas na família & \multicolumn{2}{|c|}{$4,6 \pm 1,6$} & \multicolumn{2}{|c|}{$4,2 \pm 1,3$} & 0,024 \\
\hline Número de cômodos & \multicolumn{2}{|c|}{$4,8 \pm 1,9$} & \multicolumn{2}{|c|}{$5,5 \pm 2,3$} & 0,004 \\
\hline \multirow{2}{*}{ Variáveis } & \multicolumn{2}{|c|}{ ESF } & \multicolumn{2}{|c|}{ Outros serviços } & \multirow{2}{*}{$p$ * } \\
\hline & $\mathrm{n}$ & $\%$ & $\mathrm{n}$ & $\%$ & \\
\hline \multicolumn{6}{|l|}{ Cuidador principal } \\
\hline Mãe & 242 & 89,0 & 68 & 87,2 & 0,660 \\
\hline Outros & 30 & 11,0 & 10 & 12,8 & \\
\hline Classe econômica & & & & & $<0,001$ \\
\hline Classe A & 0 & 0,0 & 2 & 2,6 & \\
\hline Classe B & 8 & 3,0 & 15 & 19,2 & \\
\hline Classe C & 194 & 72,7 & 52 & 66,7 & \\
\hline Classe D & 53 & 19,9 & 8 & 10,3 & \\
\hline Classe E & 12 & 4,4 & 1 & 1,3 & \\
\hline Escolaridade materna (anos) & & & & & 0,007 \\
\hline$\leq 8$ & 143 & 52,8 & 27 & 35,1 & \\
\hline$>8$ & 128 & 47,2 & 50 & 64,9 & \\
\hline Escolaridade paterna (anos) & & & & & 0,050 \\
\hline$\leq 8$ & 143 & 52,6 & 34 & 43,6 & \\
\hline$>8$ & 107 & 39,3 & 43 & 55,1 & \\
\hline
\end{tabular}

*Teste " $\mathrm{t}$ " para medias e teste do qui-quadrado para proporções; ESF= Estratégia Saúde da Família.

fatórios $(\geq 6,6)$ para os atributos da longitudinalidade e integralidade (ações de promoção e prevenção).

As médias dos escores dos atributos da integralidade (serviços complementares disponíveis), da orientação familiar e da orientação comunitária das ESFs alcançaram escores superiores quando comparados aos obtidos outros modelos de atenção, tendo a diferença sido estatísticamente significante.

$\mathrm{Na}$ análise conjunta dos atributos, os valor do escore geral foi significativamente mais elevado nas crianças que tinham a ESF como fonte regular de atenção à saúde do que aquelas que utilizavam outros tipos de serviço.

A Tabela 4 mostra os resultados da análise das características socioenonomicas e de utilização dos modelos de atenção relacionadas ao escore geral dos atributos obtidos. A partir dos resultados dessa tabela, tomando-se todas as variáveis com nível de significância de até $25 \%(p<0,25)$, realizou-se uma análise de regressão múltipla (Regressão de Poisson) para definir um modelo explicativo final. Esse modelo ficou definido pelas variáveis "serviço fonte regular de atenção" ( $\mathrm{RP}=1,24$ e IC95\%=1,11-1,39) e "número de atendimentos" ( $\mathrm{RP}=1,13$ e IC95\%=1,02$1,25)$, revelando que os cuidadores das crianças assistidas pela ESF e que já haviam realizado mais de dez atendimentos atribuíam melhores escores aos locais de referência. 
Características de saúde e utilização dos serviços de saúde de crianças menores de dois anos de idade, cadastradas nas equipes da Estratégia Saúde da Família, segundo o tipo de serviço referido pelo cuidador como fonte regular de atenção à saúde. Montes Claros, MG, 2009.

\begin{tabular}{|c|c|c|c|c|c|}
\hline \multirow{2}{*}{ Variáveis } & \multicolumn{2}{|c|}{ ESF } & \multicolumn{2}{|c|}{ Outros } & \multirow{2}{*}{$p$ * } \\
\hline & $\mathrm{n}$ & $\%$ & $\mathrm{n}$ & $\%$ & \\
\hline Crianças que consultaram com especialista & 67 & 25,2 & 19 & 24,4 & 0,882 \\
\hline Crianças com patologias crônicas & 17 & 6,3 & 13 & 16,7 & 0,013 \\
\hline Tempo atendimento pelo serviço & & & & & 0,187 \\
\hline Menos de 6 meses & 50 & 18,4 & 21 & 26,9 & \\
\hline 6 a 12 meses & 99 & 36,4 & 22 & 28,2 & \\
\hline 1 a 2 anos & 123 & 45,2 & 35 & 44,9 & \\
\hline $\mathrm{N}^{\circ}$ de consultas realizadas no local & & & & & 0,070 \\
\hline$\leq 10$ & 161 & 59,2 & 55 & 70,5 & \\
\hline$>10$ & 111 & 40,8 & 23 & 29,5 & \\
\hline Motivo pelo qual escolheu o local & & & & & $<0,001$ \\
\hline Você ou alguém de sua família o escolheu & 60 & 22,1 & 58 & 74,4 & \\
\hline Você foi designado a ele & 209 & 76,8 & 17 & 21,8 & \\
\hline Outro motivo & 3 & 1,1 & 3 & 3,8 & \\
\hline
\end{tabular}

*Teste do qui-quadrado para proporções; ESF= Estratégia Saúde da Família.

\section{Tabela 3}

Média dos Escores dos atributos da APS, identificados pelos cuidadores de crianças menores de dois anos de idade, cadastradas nas equipes da Estratégia Saúde da Família, segundo o tipo de serviço referido como fonte regular de atenção à saúde das crianças. Montes Claros, MG, 2009.

\begin{tabular}{|c|c|c|c|}
\hline \multirow{2}{*}{ Atributos da APS } & ESF & Outros & \multirow[t]{2}{*}{$p^{*}$} \\
\hline & $\bar{X} \pm \mathrm{DP}$ & $\bar{X} \pm D P$ & \\
\hline Primeiro contato & $5,4 \pm 1,8$ & $5,5 \pm 2,0$ & 0,663 \\
\hline Longitudinalidade & $8,2 \pm 1,5$ & $8,0 \pm 1,4$ & 0,428 \\
\hline Coordenação & $6,3 \pm 3,4$ & $7,7 \pm 2,8$ & 0,920 \\
\hline Integralidade - serviços básicos disponíveis & $5,6 \pm 3,4$ & $5,8 \pm 3,9$ & 0,558 \\
\hline Integralidade - serviços complementares disponíveis & $4,9 \pm 1,9$ & $4,0 \pm 2,5$ & 0,001 \\
\hline Integralidade - ações de promoção e prevenção recebidos & $8,0 \pm 2,8$ & $7,8 \pm 3,2$ & 0,575 \\
\hline Orientação familiar & $4,3 \pm 2,6$ & $3,5 \pm 2,4$ & 0,009 \\
\hline Orientação comunitária & $5,6 \pm 2,5$ & $1,3 \pm 2,0$ & $<0,001$ \\
\hline Essencial & $6,9 \pm 1,1$ & $6,8 \pm 1,1$ & 0,438 \\
\hline Derivado & $5,0 \pm 2,1$ & $2,4 \pm 1,7$ & $<0,001$ \\
\hline Geral & $6,4 \pm 1,2$ & $5,7 \pm 1,1$ & $<0,001$ \\
\hline
\end{tabular}

*Teste "t" para medias; \#Diferença estatisticamente significativa $(p<0,05)$ entre Estratégia Saúde da Família e outros serviços; ESF= Estratégia Saúde da Família; APS= Atenção Primária à Saúde. 
Características sociodemográficas e de utilização dos serviços de saúde classificados de crianças menores de dois anos de idade, cadastradas nas equipes da Estratégia Saúde da Família, segundo a percepção do cuidador sobre o escore geral da Atenção Primária à Saúde. Montes Claros, MG, 2009.

\begin{tabular}{|c|c|c|c|c|c|}
\hline \multirow{2}{*}{ Variáveis } & \multicolumn{2}{|c|}{ Alto escore geral $(\geq 6,6)$} & \multicolumn{2}{|c|}{ Baixo escore geral $(<6,6)$} & \multirow{2}{*}{$p$ * } \\
\hline & $\mathrm{n}$ & $\%$ & $\mathrm{n}$ & $\%$ & \\
\hline Idade das crianças (meses) & & & & & 0,793 \\
\hline$<12$ & 66 & 47,1 & 96 & 45,7 & \\
\hline $12-23$ & 74 & 52,9 & 114 & 54,3 & \\
\hline Idade das mães (anos) & & & & & 0,194 \\
\hline$\leq 25$ & 78 & 55,7 & 98 & 46,9 & \\
\hline$>25$ & 62 & 44,3 & 111 & 53,1 & \\
\hline Idade dos pais (anos) & & & & & 0,543 \\
\hline$\leq 25$ & 46 & 33,3 & 61 & 29,9 & \\
\hline$>25$ & 92 & 66,7 & 143 & 70,1 & \\
\hline $\mathrm{N}^{\circ}$ irmãos & & & & & 0,531 \\
\hline Até 1 & 97 & 69,3 & 152 & 74,4 & \\
\hline$\geq 2$ & 43 & 30,7 & 58 & 27,6 & \\
\hline Sexo da criança & & & & & 0,694 \\
\hline Masculino & 71 & 50,7 & 102 & 48,6 & \\
\hline Feminino & 69 & 49,3 & 108 & 51,4 & \\
\hline Cuidador principal & & & & & 0,493 \\
\hline Mãe & 126 & 90,0 & 184 & 87,6 & \\
\hline Outros & 14 & 10,0 & 26 & 12,4 & \\
\hline Profissão do pai & & & & & 0,224 \\
\hline Qualificado & 95 & 70,9 & 131 & 64,5 & \\
\hline Não qualificado & 39 & 29,1 & 72 & 35,5 & \\
\hline Critério Brasil & & & & & 0,670 \\
\hline$A B$ & 8 & 5,8 & 17 & 8,2 & \\
\hline $\mathrm{C}$ & 101 & 73,2 & 145 & 70,0 & \\
\hline $\mathrm{DE}$ & 29 & 21,0 & 45 & 21,7 & \\
\hline Escolaridade materna (anos) & & & & & 0,051 \\
\hline$\leq 8$ & 59 & 42,4 & 111 & 53,1 & \\
\hline$>8$ & 80 & 57,6 & 98 & 46,9 & \\
\hline Escolaridade paterna (anos) & & & & & 0,149 \\
\hline$\leq 8$ & 64 & 49,2 & 113 & 57,4 & \\
\hline$>8$ & 66 & 50,8 & 84 & 42,6 & \\
\hline Serviço fonte regular de atenção & & & & & $<0,001$ \\
\hline Saúde da Família & 122 & 87,1 & 150 & 71,4 & \\
\hline Outros & 18 & 12,9 & 60 & 28,6 & \\
\hline $\mathrm{N}^{\circ}$ de consultas realizadas no local & & & & & 0,020 \\
\hline$\leq 10$ & 76 & 54,3 & 140 & 66,7 & \\
\hline$>10$ & 64 & 45,7 & 70 & 33,3 & \\
\hline
\end{tabular}

*Teste do qui-quadrado para proporções. 
Para uma análise específica das crianças cujos cuidadores referiam a ESF como fonte regular de atenção $(n=272)$, buscou-se conhecer as características que pudessem estar associadas com melhor avaliação da estratégia, através do alto escore geral da APS (Tabela 5). Apenas a escolaridade materna mostrou-se estatisticamente associada a maiores escores.

\section{Tabela 5}

Características sociodemográficas e de utilização dos serviços de saúde classificados com alto e baixo escore geral da APS entre as crianças que tem a ESF como fonte regular de atenção. Montes Claros, MG, 2009.

\begin{tabular}{|c|c|c|c|c|c|}
\hline \multirow{2}{*}{ Variáveis } & \multicolumn{2}{|c|}{$\begin{array}{l}\text { Alto escore geral da } \\
\qquad \operatorname{APS}(\geq 6,6)\end{array}$} & \multicolumn{2}{|c|}{$\begin{array}{l}\text { Baixo escore geral da } \\
\qquad \text { APS }(<6,6)\end{array}$} & \multirow{2}{*}{$p^{*}$} \\
\hline & $\mathrm{n}$ & $\%$ & $\mathrm{n}$ & $\%$ & \\
\hline Idade das crianças (meses) & & & & & 0,636 \\
\hline$<12$ & 58 & 47,5 & 67 & 44,7 & \\
\hline $12-23$ & 64 & 52,5 & 83 & 55,3 & \\
\hline Idade das mães (anos) & & & & & 0,577 \\
\hline$\leq 25$ & 67 & 54,9 & 77 & 51,7 & \\
\hline$>25$ & 55 & 45,1 & 72 & 48,3 & \\
\hline Idade dos pais (anos) & & & & & 0,580 \\
\hline$\leq 25$ & 42 & 35,0 & 46 & 31,7 & \\
\hline$>25$ & 78 & 65,0 & 99 & 68,3 & \\
\hline$N^{\circ}$ irmãos & & & & & 0,605 \\
\hline Até 1 & 81 & 66,4 & 104 & 69,3 & \\
\hline$\geq 2$ & 41 & 33,6 & 46 & 30,7 & \\
\hline Sexo da criança & & & & & 0,210 \\
\hline Masculino & 63 & 51,6 & 66 & 44,0 & \\
\hline Feminino & 59 & 48,4 & 84 & 56,0 & \\
\hline Profissão do pai & & & & & 0,101 \\
\hline Qualificado & 82 & 69,5 & 86 & 59,7 & \\
\hline Não qualificado & 36 & 30,5 & 58 & 40,3 & \\
\hline Profissão da mãe & & & & & 0,141 \\
\hline Qualificado & 30 & 24,6 & 26 & 17,3 & \\
\hline Não qualificado & 92 & 75,4 & 24 & 82,7 & \\
\hline Critério Brasil & & & & & 0,641 \\
\hline$A B$ & 4 & 3,3 & 4 & 2,7 & \\
\hline $\mathrm{C}$ & 90 & 75,0 & 104 & 70,7 & \\
\hline DE & 26 & 21,7 & 39 & 26,5 & \\
\hline Escolaridade materna (anos) & & & & & $0,030^{\#}$ \\
\hline$\leq 8$ & 55 & 45,5 & 88 & 58,7 & \\
\hline$>8$ & 66 & 54,5 & 62 & $(41,3)$ & \\
\hline Escolaridade paterna (anos) & & & & & 0,119 \\
\hline$\leq 8$ & 58 & 51,8 & 85 & 61,6 & \\
\hline$>8$ & 54 & 48,2 & 53 & 38,4 & \\
\hline $\mathrm{N}^{\circ}$ de consultas realizadas no local & & & & & 0,296 \\
\hline$\leq 10$ & 68 & 55,7 & 93 & 62,0 & \\
\hline$>10$ & 54 & 44,3 & 57 & 38,0 & \\
\hline
\end{tabular}

*Teste do qui-quadrado para proporções; \#Diferença estatisticamente significativa $(p<0,05)$ entre Estratégia Saúde da Família e outros serviços; APS= Atenção Primária à Saúde. 


\section{Discussão}

No presente estudo, a avaliação dos serviços de saúde utilizando um instrumento validado permitiu aferir a percepção dos cuidadores sobre a qualidade destes serviços dirigidos à população infantil, através da medida da extensão dos atributos da APS. A identificação da ESF como serviço de referência para o cuidado de saúde das crianças foi observada para a maioria dos entrevistados no presente estudo, mostrando semelhança com pesquisas realizadas em outros locais do país. 19,20

Os achados relacionados ao perfil sociodemográfico das crianças que utilizavam a ESF e, portanto, que procuram a ESF como fonte regular de atenção, sinalizam para uma maior vulnerabilidade social e econômica. Estes resultados assemelham-se aos encontrados em outros estudos, que mostraram que os estratos mais pobres da população utilizam mais os serviços da ESF como fonte regular de assistência à saúde. 10,20,21 As famílias das crianças que usam a ESF como serviço regular de assistência referem à opção por este tipo de serviço como uma destinação a ele (devida residirem na área de abrangência) e não como uma opção da família/cuidador. Outros estudos têm avaliado os motivos que levam a população a escolher o local para acompanhamento de saúde, estando entre os mais referidos a proximidade da moradia e a qualidade do atendimento. $22,23 \mathrm{~A}$ análise global dos resultados permite inferir que os cuidadores percebem, nas equipes da ESF, diferenciais de qualidade em relação a outros serviços.

Em relação aos escores de cada atributo da APS entre os serviços identificados como fonte regular de atenção pelos cuidadores, destacam-se os baixos escores para a maioria dos atributos avaliados. Somente a longitudinalidade e a integralidade (ações de promoção e prevenção recebidos) foram bem avaliados e alcançaram alto escore $(\geq 6,6)$ para ambos os serviços.

No atributo do primeiro contato os escores observados são baixos e semelhantes entre os serviços indicando que ainda existem barreiras que dificultam o acesso das crianças aos serviços de saúde. Esse fato também foi identificado em estudos semelhantes. 8,20 É possível que as dificuldades de alcance desse atributo estejam vinculadas às particularidades da criança e às demandas curativas apresentadas aos serviços de saúde pelo maior volume de afecções agudas que apresenta. Em outras palavras, a criança adoece mais frequentemente que o adulto e principalmente por condições agudas. Esta hipótese é reforçada pelos escores mais altos observados em ambos os tipos de serviços para a dimensão de ações de promoção e prevenção recebidas por este grupo. É preciso pois, uma maior preocupação por parte dos serviços de saúde quanto à maior vulnerabilidade biológica e social da criança para programação específica e provimento de maior acesso.

A presença adequada da longitudinalidade é um fator essencial para o sistema de saúde, pois este atributo tende a produzir diagnósticos e tratamentos mais precisos, além da redução dos encaminhamentos desnecessários para especialistas e para a realização de procedimentos de maior complexidade. ${ }^{24}$ Registrou-se homogeneidade entre as ações de longitudinalidade, com valores satisfatórios em ambos os serviços. Este aspecto é bastante positivo, já que para a assistência à saúde da criança é preciso maior interação com a família e com a história de vida da criança.

A coordenação obteve valor próximo ao ideal para as equipes da ESF, não sendo percebidas diferenças nas avaliações da ESF e dos outros tipos de serviço. O valor obtido, próximo do ponto de corte proposto, sugere uma coordenação relativamente adequada, o que também está em consonância com outros estudos. 8,11

Analisando a integralidade verificou-se que a dimensão das "ações de promoção e prevenção recebidas" está efetivamente sendo executada pelos serviços de saúde, o que já demonstra uma mudança do modelo assistencial de saúde no país, onde as organizações do setor sempre dividiram suas ações em médico-assistenciais e preventivas e, por sua vez, tiveram pesos distintos no sistema sendo oferecidas de maneira desigual. 25 Em relação às dimensões da integralidade "serviços básicos disponíveis" e "serviços complementares disponíveis", observou-se valor do escore aquém do ideal, o que também foi observado no estudo de Harzheim. 20 Este fato demonstra a necessidade das unidades de saúde da ESF disponibilizarem mais serviços para atenderem às necessidades básicas de saúde da população, incluindo insumos como vacinas e medicamentos. Registra-se também a necessidade de uma melhor qualificação dos profissionais em relação aos cuidados no manejo das condições mais comuns e de grande impacto na saúde geral das famílias e da comunidade (tais como alcoolismo, uso de drogas, problemas de saúde mental, entre outros). ${ }^{26}$ Torna-se necessário pois a ampliação do escopo de atuação dos serviços de saúde, conforme já apontado por Harzheim. ${ }^{20}$

Espera-se que elevados escores de longitudinalidade sejam acompanhados também de escores mais elevados para o atributo de orientação familiar, já que, conforme referido, a assistência à saúde infantil 
pressupõe maior interação com a família. Contudo, no presente estudo, verificou-se que o escore do atributo da orientação familiar não apresentou valor satisfatório em nenhum dos serviços avaliados, apesar de elevados escores de longitudinalidade. Destaca-se, porém que houve diferença estatística entre os escores dos serviços, e as equipes de saúde da família foram mais bem avaliadas em relação aos demais serviços, o que também foi confirmado em estudos semelhantes. $8,10,11,20$ Registra, assim, a que a ESF tende a propiciar, de fato, uma mudança no modelo assistencial, que passa a valorizar mais o contexto de vida das pessoas e a inserção do sujeito na família e na comunidade. Este segundo aspecto é, notadamente destacado pelos cuidadores das crianças, que atribuíram melhores escores para o atributo da orientação comunitária à equipes da ESF. A diferença, estatisticamente significativa observada neste estudo também foi confirmada por outros autores. 10,11,20 Alguns fatores parecem estar relacionados com a melhor efetividade da orientação comunitária na ESF, como as ações de visita domiciliar realizadas por todos os profissionais, mas principalmente pelos agentes comunitários de saúde, o que facilita a vigilância à saúde e o acompanhamento das famílias da comunidade.

Ao se analisar o valor do escore essencial, percebeu-se que os serviços de saúde referidos pelos cuidadores como fonte regular de atenção à saúde da criança estão fornecendo atenção em consonância com os atributos essenciais da APS. Em relação ao valor do escore derivado ainda é necessário que os serviços de saúde aprimorem as ações que têm como foco a família e a comunidade, mas é importante enfatizar que a ESF está com valor do escore derivado acima do dobro em relação aos outros serviços. A análise conjunta dos atributos - escore geral - registra que a ESF está próximo do valor referido como ideal, e apresenta diferença superior em relação aos outros tipos de serviço.

Um dado relevante neste estudo se deve ao fato de que os atributos da APS foram avaliados segundo a percepção dos usuários (cuidadores), e estes tendem a avaliar os serviços de saúde de maneira menos favorável do que quando são avaliados pelos profissionais que atuam no serviço. 10,11 É possível, portanto, que uma abordagem ampliada do processo avaliativo dos atributos da APS registre melhores escores com a inserção de novos atores sociais.

Os resultados mostraram ainda que utilizar a ESF como fonte regular de atenção e ter realizado mais consultas no serviço de referência ( $>10$ vezes) são variáveis que estão associadas à melhor percepção do serviço (alto escore geral da APS).
Seguramente existe a percepção de que se trata de serviços diferenciados em relação aos cuidados com a família e com a comunidade. Em contrapartida, as famílias que utilizam outros serviços percebem a assistência desarticulada em relação aos atributos da APS, ainda que se reconheçam aspectos positivos da referida assistência. Sobre o número elevado de consultas, este traduz, para crianças menores de dois anos de idade, maior contato dos cuidadores com os profissionais dos serviços de saúde e maior integração aos mesmos serviços. É natural, pois esperar, para esses cuidadores uma percepção mais positiva dos cuidados recebidos.

Apenas a escolaridade materna maior que oito anos mostrou-se estatisticamente associada com melhores escores atribuídos a ESF para o grupo de cuidadores que utiliza apenas esse serviço como fonte regular de atenção à saúde. Não foram identificados estudos com resultados semelhantes na literatura nacional. Contudo, a pesquisa realizada por Ibañez et al. ${ }^{9}$ que comparou os escores dos atributos da APS entre grupos de municípios que tinham indicadores sociais menos favoráveis com grupos que tinham estes indicadores mais favoráveis, verificou uma associação positiva entre melhores indicadores sociais e maiores escores atribuídos a ESF. Embora não seja possível fazer uma inferência precisa sobre esse achado, é possível indagar se esse dado não traduz um reconhecimento de maior efetividade dos serviços da ESF por parte das mães com maior escolaridade.

Os presentes resultados devem ser interpretados considerando algumas limitações. O estudo apresenta apenas a visão do usuário, o que indica uma avaliação importante dentro do processo assistencial, pois é quem efetivamente experimenta o serviço, em seus aspectos positivos e negativos. Ocasionalmente esta pode ser a uma visão mais crítica do que aquela realizada por outros atores sociais. Também é preciso destacar que os resultados são restritos a um único município. Todavia, a carência de estudos nacionais sobre o tema aponta para a relevância de avaliações similares.

Foi possível concluir com este estudo que o PCATool infantil representa um bom instrumento de avaliação e comparação da estrutura e processo dos serviços de APS dirigidos à população infantil. Destaca-se a expansão de sua utilização em outros países do mundo. ${ }^{27-30}$ A APS de qualidade é uma estratégia efetiva na busca de maior promoção da saúde, prevenção de doenças, melhor estado de saúde das crianças e maior satisfação dos cuidadores. A literatura internacional é rica em evidências de mostram que os sistemas de saúde que 
possuem uma APS fortalecida são mais efetivos. 12 Reconhecendo que a ESF é o espaço preferencial para a promoção da saúde infantil no âmbito sanitário, os resultados deste estudo apontam para a necessidade de melhoria de alguns atributos da

\section{Referências}

1. Tejada de Rivero DA. Alma-Ata: 25 años después. Rev Perspectiva de Salud. 2003; 8: 2-7.

2. Almeida C. Eqüidade e reforma setorial na América Latina: um debate necessário. Cad Saúde Pública. 2002; 18: 23-36

3. Malta DC, Santos FP. O programa de saúde da família (PSF) e os modelos de assistência à saúde no âmbito da reforma sanitária brasileira. Rev Med Minas Gerais 2003 13: 251-9.

4. Brasil. Ministério da Saúde. Departamento de Atenção Básica. Programa Saúde da Família. Rev Saúde Pública. 2000; 34: 316-9.

5. Brasil. Portaria $n^{\circ}$ 648. Aprova a Política Nacional de Atenção Básica, estabelecendo a revisão de diretrizes e normas para organização da Atenção Básica para o Programa Saúde da Família (PSF) e o Programa Agentes Comunitários de Saúde (PACS). Diário Oficial da União. 2006; 29 mar

6. Organização Pan-Americana da Saúde. Renovação da atenção primária em saúde nas Américas. Washington DC: Organização Mundial da Saúde; 2005.

7. Starfield B. Primary care: is it essential? Lancet. 1994; 344 1129-33.

8. Macinko J, Almeida C, Oliveira ES, Sá PK. Organization and delivery of primary health care services in Petrópolis, Brazil. Int J Health Plann Manage. 2004; 19: 303-17.

9. Ibañez N, Rocha JSY, Castro PC, Ribeiro MCSA, Forster AC, Novaes MHD, Viana ALD. Avaliação do desempenho da atenção básica no Estado de São Paulo. Ciênc Saúde Coletiva. 2006; 11: 683-703.

10. Elias PE, Ferreira CW, Alves MCG, Cohn A, Kishima V, Escrivão Junior A, Gomes A, Bousquat A. Atenção básica em saúde: comparação entre PSF e UBS por estrato de exclusão social em São Paulo. Ciênc Saúde Coletiva. 2006; 11: 633-41.

11. Van Stralen CJ, Belisário AS, Van Stralen TBS, Lima, AMD, Massote AW, Oliveira CL. Percepção dos usuários e profissionais de saúde sobre atenção básica: comparação entre unidades com e sem saúde da família na Região Centro-Oeste do Brasil. Cad Saúde Pública. 2008; 24 (Supl. 1): $148-58$.

12. Starfield B. Atenção primária: equilíbrio entre necessidades de saúde, serviços e tecnologia. Brasília: UNESCO/Ministério da Saúde; 2002.

13. Macinko J, Harzheim E. Instrumento de avaliação da atenção primária - sua utilização no Brasil. Rev Bras Saúde Fam. 2007; 14: 4-7.

14. Harzheim E, Stein AT, Álvarez-Dardet C. A efetividade dos atributos da atenção primária sobre a saúde infantil. Bol Saúde. 2004; 18: 23-40.

15. Shi L, Starfield B, Jiahong X. Validating the adult primary care assessment Tool. J Fam Pract. 2001, 50:161-75. prática assistencial. Essa melhoria implica em reformulações de alguns aspectos de sua estrutura e processo para oferecerem APS na qualidade que se propõe.

16. Cassady CE, Starfield B, Hurtado MP, Berk RA, Nanda JP, Friedenberg LA. Clinical care for children: measuring consumer experiences with primary care. Pediatrics. 2000; 105: 998-1003.

17. Harzheim E, Starfield B, Rajmil L, Álvarez-Dardet C, Stein AT. Consistência interna e confiabilidade da versão em português do Instrumento de Avaliação da Atenção Primária (PCATool-Brasil) para serviços de saúde infantil. Cad Saúde Pública. 2006; 22: 1649-59.

18. Oliveira MMC. Presença e extensão dos atributos da Atenção Primária à Saúde entre os serviços de Atenção Primária à Saúde em Porto Alegre: uma análise agregada. [dissertação]. Porto Alegre: Universidade Federal do Rio Grande do Sul; 2007.

19. Ribeiro LCC. Acolhimento as crianças na atenção primaria à saúde: um estudo sobre a postura dos profissionais das equipes de saúde da família. [dissertação]. Belo Horizonte: Universidade Federal de Minas Gerais; 2009.

20. Harzheim E. Evaluación de la atención a la salud infantil del Programa Saúde da Família en la región sur de Porto Alegre, Brasil. [tese]. Alicante (Espanha): Universidade de Alicante; 2004

21. Silva NN, Pedroso LC, Puccini RF, Furlani WJ. Desigualdades sociais e uso de serviços de saúde: evidências de análise estratificada. Rev Saúde Pública. 2000; 34: 44-9

22. Santos IS, Baroni RC, Minotto I, Klumb AG. Critérios de escolha de postos de saúde para acompanhamento pré-natal em Pelotas, RS. Rev Saúde Pública. 2000, 34: 603-9.

23. Ramos DD, Lima MADS. Acesso e acolhimento aos usuários em uma unidade de saúde de Porto Alegre, Rio Grande do Sul, Brasil. Cad Saúde Pública. 2003; 19: 27-34.

24. Gérvas J, Fernández MP. El fundamento científico de la función de filtro del médico general. Rev Bras Epidemiol. 2006; 9: 147-9.

25. Campos CEA. O desafio da integralidade segundo as perspectivas da vigilância da saúde e da saúde da família. Ciênc Saúde Coletiva. 2003; 8: 569-84

26. Noronha MGRCS, Cardoso OS, Moraes TNP, Centa ML. Resiliência: nova perspectiva na promoção da saúde da família? Ciênc Saúde Coletiva. 2009; 14: 497-506.

27. Pasarin MI, Berra S, Rajmil L, Solans M, Borrell C, Starfield B. [An instrument to evaluate primary health care from the population perspective]. Aten Primaria. 2007; 39: 395-401.

28. Haggerty JL, Pineault R, Beaulieu MD, Brunelle Y, Gauthier J, Goulet F, Rodrigue J. Practice features associated with patient-reported accessibility, continuity, and coordination of primary health care. Ann Fam Med. 2008; 6: 116-23. 
29. Lee JH, Choi YJ, Sung NJ, Lee JH, Choi YJ, Sung NJ, Kim SY, Chung SH, Kim J, Jeon TH, Park HK. Development of the Korean primary care assessment tool--measuring user experience: tests of data quality and measurement performance. Int J Qual Health Care. 2009; 21: 103-11.
30. Pongpirul K, Starfield B, Srivanichakorn S, Pannarunothai S. Policy characteristics facilitating primary health care in Thailand: a pilot study in transitional country. Int J Equity Health. 2009; 8:8.

Recebido em 29 de abril de 2010

Versão final apresentada em 5 de abril de 2011

Aprovado em 15 de abril de 2011 